\title{
A REORBITER FOR LARGE GEO DEBRIS OBJECTS USING ION BEAM IRRADIATION
}

\section{Shoji Kitamura, Yukio Hayakawa, Satomi Kawamoto}

Japan Aerospace Exploration Agency, Japan

In recent years, space debris problems have become very serious. The worst case occurs in the low Earth orbit (LEO) region, where debris-to-debris collisions generate new debris. The situation in the geostationary orbit (GEO) region is not as bad as that in the LEO. The debris problem in the GEO region, however, should not be left as it is because the GEO is unique and has few debris-cleansing modes. Thus, we proposed a concept for a reorbiter to reorbit large GEO debris objects such as satellites and rocket upper stages left in orbit after the ends of their missions. This concept is based on the idea of thrusting a debris object by irradiating it with an ion beam. The reorbiter, equipped with two ion engines, approaches a debris object, and the ion beam exhausted from one of the ion engines irradiates and thrusts it to change its orbit. The other engine on the opposite side is operated so that the reorbiter follows the debris object. Their orbits are raised in a spiral to a disposal orbit approximately $300 \mathrm{~km}$ higher. After that, the reorbiter returns to GEO to approach another debris object. This system can operate without catching debris objects; thus, it can be applied to a wide range of debris objects without regard to their shapes or rotations. A mission scenario was made to conduct efficient maneuvers. In the GEO region, a number of debris objects are distributed on orbit planes close to each other, and they can be reorbited one after another using a single reorbiter. For a typical model mission, the mission time and the total impulse of the ion engines were calculated. The results show that six debris objects can be reorbited in 170 days. The reorbiter has a targeted launch mass of $2500 \mathrm{~kg}$ and $6.9 \mathrm{~kW}$ of total power. The ion beam convergence, the effects of ion beam irradiation, and non-cooperative rendezvous were recognized as the critical issues of this system. A highly converged beam is required to make efficient debris irradiation. Numerical calculations and basic experiments gave a feasibility of the required irradiation efficiency of over $25 \%$. The irradiation of debris objects may cause sputtering of their surfaces and depositions of the back-sputtered materials on the reorbiter surface. The data were obtained experimentally to evaluate the effects of the depositions, especially on solar cells. The results indicated no serious contamination problems. Preliminary studies were conducted on the approach to an uncollaborative object and the maintenance of the separation distance.

\section{INTRODUCTION}

The population of space debris is increasing year by year, and debris mitigation has become essential for sustaining space activities in the future. The space debris mitigation guidelines of the Inter-Agency Space Debris Coordination Committee (IADC) recommend that spacecraft in the LEO region should be deorbited within 25 years after the ends of their missions. They also recommend that spacecraft in the GEO region should be reorbited to a disposal orbit approximately $300 \mathrm{~km}$ higher than the GEO after the ends of their missions.

However, these mitigation measures alone are not sufficient because in these orbit regions, there is already a very large population of spacecraft left after the ends of their missions. In particular, recent numerical simulations of the evolution of the space debris population indicate that the population in the crowded LEO region has reached the point where the environment is unstable and that debris population growth is inevitable because of mutual collisions between debris objects. This result suggests that debris remediation measures should be taken urgently; thus, active debris removal has begun to be widely studied.

In the GEO region, the debris population increase due to collisional cascading has not been predicted yet, although accidental break-ups from stored energy have been reported. In this region, however, unique features and problems exist as follows ${ }^{1}$.

a) The GEO is the one limited resource that the whole world utilizes. Thus, if the debris population is excessively increased by collisions or explosions, its influence will be extremely large.

b) Debris objects in the GEO region will stay there permanently because we cannot expect any decrease in the debris population because of atmospheric drag.

c) Although the sizes of the catalogued objects in the GEO region are approximately $50 \mathrm{~cm}$ or larger, there are many debris objects of smaller sizes $(20 \mathrm{~cm}$ to $30 \mathrm{~cm})$ whose exact orbits are not known. 
d) To date, not all geosynchronous satellites have been reorbited after the ends of their missions in compliance with the IADC guidelines. Thus, the number of large objects in this region is increasing.

For these reasons, it is becoming more and more important to protect the GEO region from debris generation.

The situation of space objects in the GEO region is as follows ${ }^{2}$. More than 1,300 objects are catalogued in and near the GEO. Although reorbiting compliant with the IADC reorbiting guidelines has been conducted to a certain extent, technical difficulties remain in conducting orbit raising at the end of missions, and there are some cases where reorbiting cannot be conducted because of satellite failure. The end-of-life reorbiting rate of geosynchronous satellites has been $60 \%$ on average for the past eight years. In 2011, 16 spacecraft reached the end of their life. Only 13 of them were reorbited in compliance with IADC rules. This observation shows that there are still too many satellites that were not or could not be properly reorbited.

Recently, in these situations, proposals have been actively made for reorbiting the satellites left as space debris after the end of their life. In Europe, the development of a debris removal system called ROGER has begun ${ }^{3}$. The system uses a net to catch GEO debris objects and transport them to the disposal orbit. In the US, a GEO debris reorbiter called GLiDeR has been proposed, which would generate electrostatic force to tug large debris objects for reorbit $^{4}$.

In Japan, we proposed a new concept for a reorbiter that reorbits large GEO debris objects to the disposal orbit using the thrust of ion beam irradiation ${ }^{5,6}$. The same concept was also proposed by other researchers, but their interest is in deorbiting debris objects in the LEO region ${ }^{7,8}$.

Since the proposal, we have been conducting a system study and numerical and experimental studies on some issues critical to realizing this system. This paper describes the results of these studies, specifically on the concept of the reorbiter, the mission and the reorbiter system and the issues that have to be addressed such as the ion beam convergence, the effects of ion beam irradiation, and non-cooperative rendezvous.

\section{CONCEPT OF THE REORBITER}

\section{II.I Concept and the Features}

Figure 1 shows a schematic drawing of the reorbiter concept. The reorbiter is equipped with two (or more) ion engines (ion engines A and B) whose thrust directions are opposite. Ion engine A is installed on the side of the reorbiter that faces the debris object and ion engine $\mathrm{B}$ on the opposite side of the reorbiter. The reorbiter approaches a large debris object assumed to be a dead satellite or rocket upper stage within a certain distance. The ion beam exhausted continuously from ion engine A irradiates the debris object and applies thrust to it. This thrust raises the orbit of the debris object gradually. The thrust is so small that the orbit is raised in a spiral manner. Ion engine B is operated such that the reorbiter follows the debris object by its thrust. The thrusts of the ion engines are adjusted such that the distance between the debris and the reorbiter is kept within a certain range. After they reach the disposal orbit, the reorbiter returns to the GEO region to reorbit another debris object, and this round trip is repeated to reorbit a number of debris objects.

This reorbiter features the following.

a) Technologies for capturing debris objects are not required.

b) This method of reorbiting does not depend on the detailed shapes of the debris objects, such as grappled parts. Thus, similar procedures can be used for different debris objects.

c) It is not necessary to reduce the rotation or the tumbling of debris objects. This is because the velocity of ions is extremely high (about $38 \mathrm{~km} / \mathrm{s}$ for xenon ions at $1-\mathrm{kV}$ beam potential for example). Even if the debris objects are rotating or tumbling and their surfaces are moving, these moving velocities are negligibly small compared with the ion velocity, and so the momentum given to the debris objects are not changed effectively 
both in the magnitude and direction. Thus, effectively the same thrusts to the debris objects are expected as they are at rest.

The velocity increment is approximately $11 \mathrm{~m} / \mathrm{s}$ for reorbiting a GEO debris object to an orbit $300 \mathrm{~km}$ higher than GEO. It was calculated from the difference between the orbital velocities at these two orbits because this orbit transfer is by low thrusting. This velocity increment is much smaller than the velocity increment of $90 \mathrm{~m} / \mathrm{s}$ to $140 \mathrm{~m} / \mathrm{s}$ required for deorbiting a LEO debris object to a lower orbit that complies with the " 25 -year rule" in the debris mitigation guidelines.

The velocity increment of $11 \mathrm{~m} / \mathrm{s}$ is small enough to be provided by ion beam irradiation. Using this measure, we can avoid applying some of the difficult technologies required for active debris removal in the LEO. Specifically, we can avoid reducing tumbling, or the angular momentum, of the debris object, synchronous flying around the debris object, confirming what to grapple, and capturing the debris object. The proposed reorbiter relies on a rendezvous with an uncollaborative debris target, which is also required for various methods of active debris removal.

However, there are some technical issues that should be addressed. They are the following:

a) improvement in the convergence of the ion beam exhausted from the ion engine.

b) evaluation of the effects of ion beam irradiation on the debris object and the reorbiter and of countermeasures if necessary.

c) approach to an uncollaborative object by low thrust and orbit transfer by low thrust, for which a short relative distance must be kept to the object.

\section{II.II Interactions of the Ion Beam with the Debris Object}

The interactions of the ion engine beam with the debris object are discussed in the literature ${ }^{7}$. When the debris object is irradiated by the ion beam, it receives a force that comes from the momentum of the beam ions colliding with the object. The high-velocity ions emitted from the ion engine reach the debris surface penetrating the debris material while losing their momentum and energy through collisions until they stop at a distance of typically a few nanometers from the surface. Most ions are then rejected from the material lattice, and abandon the surface at velocities in the order of the surface thermal speed (typically $0.02 \mathrm{eV}$ corresponding to $300 \mathrm{~K}$ ), orders of magnitude lower than the velocity of the ion beam (typically $1 \mathrm{keV}$ ). Thus, the contribution to momentum transfer of the backscattered particles is negligible.

The above collisional energy exchange process can cause a number of the material atoms to reach the energy needed to leave the debris surface, giving sputtering phenomena. For the case of xenon ions colliding an aluminum surface at about $1 \mathrm{keV}$, the energy of the sputtered particles is more than two orders of magnitude smaller than the energy of the impinging ions ${ }^{7}$. Thus, the effect of momentum of the sputtered material on the momentum transfer to the debris object can be neglected.

For other typical surface materials of debris objects such as glass (cover glass of solar cells) and polyimide (surface material of multilayer insulations), no data were available on the sputtering yield and energy per sputtered particle for xenon ion impingements. As the first step of the study, however, it would be reasonable to assume that almost the same situations as in the aluminum case can hold. Moreover, even if the sputtered materials have any effects on the thrust to the debris object, they would be positive in the sense that the thrust would be increased.

If we discuss the sputtering in more detail, we should consider the angular dependence of the sputtering yield. The sputtered particles have distributions with the sputtered direction. However, we assumed that its effects on the force to the debris object would be negligible because the total effects are very small as described above. Moreover, the sputtering yield has dependence on the incident angle of the colliding ions. Though slanted incidence of the col- 
liding ions can increase the sputtering yield in comparison with the case of normal incidence, the increase is no more than several times, and thus its effects are also negligible.

\section{MISSION AND REORBITER SYSTEM}

\section{III.I Candidate Objects and Sample Mission Model}

Although large GEO debris objects consist of spent rocket stages and defunct satellites, we have selected as the first candidates for reorbit rocket upper stages and spin-stabilized satellites. They have roughly cylindrical shapes; thus, it is easier to evaluate the irradiation thrust to these debris objects. Three-axis satellites, with much more complicated configurations, may be considered as targets for the next step.

To conduct efficient debris-reorbit missions, we should consider both of the total number of reorbited debris objects and their total mass in addition to their orbit regions for maximizing the risk reduction and the costeffectiveness. In this study, we assumed that a reorbiter conducts multiple reorbit missions for a certain number of debris objects. The reorbit efficiency depends on how densely the debris objects are orbiting. To examine this, the orbits of the candidate debris objects in the GEO region were plotted on a diagram of the inclination angle $\boldsymbol{i}$ versus the right ascension of the ascending node (RAAN) $\Omega$, as shown in Fig. 2. This plot shows that the objects in the GEO region are concentrated near a single curve on the $\boldsymbol{i}-\Omega$ diagram. This suggests that after the reorbit of the first debris object is finished, the reorbiter can return to the immediate vicinity of the debris object of the next target with a small velocity increment.

Considering this situation, we assumed a sample model of a reorbit mission, as shown in Table 1. The sample mission consists of the reorbit of six objects orbiting with dispersions in the inclination angles smaller than 2 deg and in the RAAN smaller than $5 \mathrm{deg}$. These parameters were selected from the following consideration. From the $i-\Omega$ diagram data in Fig. 2, we made a histogram to search the areas where debris objects are concentrated. We counted the number of the debris objects that are included in each of the $i-\Omega$ areas with 2-deg inclination width and 5-deg RAAN width. Then, we found six areas in each of which five debris objects are included and an area in which six ones are included. Thus, as the first step of the study, we selected a mission reorbiting six debris objects because six is the number of the debris objects in the densest area. This study was conducted for an assumed sample mission to avoid non-technical arguments that may be caused by dealing with existing debris objects.

\section{III.II Ion Engines}

For this reorbiter system, two types of ion engine are needed: one for irradiating debris objects and the other for orbit control. The ion engines for irradiating debris objects are required to have a highly converged ion beam for efficient irradiation. This will allow a longer separation from the debris object and will bring safer orbit control and reduction in back-sputtering effects. By using ion engines with higher ion beam convergence, we can obtain the required thrust to debris objects with smaller ion-engine thrusts. This means that the ion engines consume less electric power, and so we can conduct the same mission by using the reorbiter system with lower power capability. No special requirements are imposed on the ion engines for orbit control, so we can use conventional ones.

With the above considerations, we set the requirements for the ion engines as shown in Table 2. The total thrust of the ion engines for irradiation was chosen to be $80 \mathrm{mN}$. This choice was made by the following consideration. Obviously, lower thrust levels would make the mission periods longer, which would require longer lifetime of the reorbiter system including the ion engines, and also would bring higher operation cost. Contrarily, higher thrust levels would require larger electric power for the ion engines, and so larger power capability of the reorbiter system. It is difficult to conduct quantitative optimization of the system including the ion engines at this stage of the research because there are a lot of unknown factors. Thus, first we assumed plausible values for the related parameters; 80 
$\mathrm{mN}$ for the total thrust of the ion engines for debris irradiation, $25 \%$ for the irradiation efficiency (the ratio of the thrusts to the debris object to the total thrust of the ion engines for debris irradiation), $2000 \mathrm{~kg}$ for the debris object mass. Then, with these values and $\Delta \mathrm{V}$ of $11 \mathrm{~m} / \mathrm{s}$, we obtained 12.6 days for the orbit raising time. Because these seemed acceptable, we proceeded further with this choice of the total thrust of the ion engines for irradiation.

The total thrust of the ion engines for orbit control can be determined from the sum of two forces; the force counteracting the total thrust of the ion engines for irradiation, and the force providing the same value of acceleration to the reorbiter as that to the debris object to maintain constant separation from it. This imposes the minimum requirement for the total thrust of the ion engines for orbit control. As a design requirement, however, the total thrust of 160 $\mathrm{mN}$ by four $40-\mathrm{mN}$ thrusters was chosen, as shown in Table 2, from a different reason. In the descending orbit transfer, the ion engines for debris irradiation are dormant, and thus the electric power that these ion engines would consume if operated can be allotted to the ion engines for orbit control to produce more thrust. By doing so, the time required to transfer to the next debris object can be shortened. Note that it is assumed that, while returning to the next target, the reorbiter takes the reverse attitude around its yaw axis with the ion engines for orbit control headed forward.

We assumed a lower performance for the ion engines for debris irradiation because the ion beam convergence should have the higher priority. For the reorbiter system study, the performance of the ion engine was simply defined as the thrust-to-power ratio of the whole ion engine system. As will be described in Section IV.I, the reduction in the beam divergence can be realized by operating the ion engine with low ion number density in the discharge chamber, and also by extracting the ion beam through the grids at not fully maximized conditions. Such operating conditions may lower the ion engine performance.

One of the important issues in ion engine applications is thruster lifetime, or more specifically grid lifetime. In this reorbiter system, ion engines with low beam divergence are required for irradiating the debris object efficiently. However, no matter whether the beam divergence is low or high, the principle of ion engine operation is the same, and so the main mechanism of grid erosion is expected to be the same (mainly sputtering by charge exchange ions). Thus, the grid lifetime in low-divergence operation is expected to be of the same order of magnitude as the one in conventional operation, or at least, not extremely shorter. As will be shown in Section III.III, the total operation time of the ion engines for debris irradiation is much less than that of the ion engines for orbit control. This is because the former ones are used only for orbit raising while the latter ones are used also for returning to the GEO region for the next target in addition to orbit raising. This indicates that the lifetime requirement for the ion engine for debris irradiation is much lower than that for the ion engine for orbit control. Moreover, as far as this sample mission is concerned, lifetimes of the both ion engines may not be an issue since the $\Delta \mathrm{V}$ requirement is low.

\section{III.III Mission Outline}

For the sample mission model assumed in III.I and the ion engines shown in Table 2, we calculated the velocity increments required for reorbiting the debris objects.

In the calculation, we included the spiral circular orbit transfers, the circular-to-elliptic orbit transfers and vice versa, the inclination changes, and the RAAN adjustments. In the spiral circular orbit transfers, the thrust is continuously applied in the tangential direction of the orbit. In the circular-to-elliptic orbit transfers and vice versa, the ion engines are operated for four hours in total before and after each of the points where thrusting would be needed in the orbit transfers by impulsive maneuvers. In this case, it is taken into account that the decrease in efficiency would be about 3\%. In the inclination changes and the RAAN adjustments, the ion engines are also operated for four hours in total on the same conditions as in the above case. In this case, it is taken into account that the decrease in efficiency 
would be about $5 \%$. In returning to the next target object, the maneuvers changing the orbit plane are conducted first, and followed by the maneuvers in the orbit plane.

The initial reorbiter mass was assumed to be $1500 \mathrm{~kg}$, and the distance between the reorbiter and the debris object was $20 \mathrm{~m}$. This assumption of $20 \mathrm{~m}$ was made by the following consideration. The candidate objects of rocket upper stages and spin-stabilized satellites have the lengths of about $7 \mathrm{~m}$ in the relatively large cases. A few times of these lengths would be a reasonable assumption for the separation distance between the reorbiter and the debris object. Shorter separation distances would increase the collision risk of the reorbiter to the debris object, and longer ones would decrease the irradiation efficiency because of the ion beam divergence.

As will be described in Chapter VI, this reorbiter is assumed to be equipped with a rendezvous sensor camera and laser sensors for navigation to maintain the relative separation to the debris object. Under some conditions during the ascending maneuvers, the rays of the sun may directly enter the rendezvous sensor camera facing the debris object, and the function of the camera may be restricted. To cope with this problem, we assumed the ion engine operation stops while this is happening. To keep the ascending orbit near circular, we also assumed the ion engine operation stops for the symmetrical part of the orbit.

The calculation was conducted with the above assumptions. Figure 3 shows the total impulse produced by the ion engines for orbit control and for debris irradiation versus the required mission time. Table 3 shows the required propellant mass as well as the total impulse. In Fig. 3, the sloped segments of the total impulse for debris irradiation correspond to the orbit rising to the disposal orbit and the horizontal segments to the return maneuvers to the GEO region. The total impulse required for orbit control is much larger than that for debris irradiation because the ion engines for orbit control have to execute the orbital maneuvers of the inclination changes and the orbit correction in addition to the ascending and descending maneuvers.

The assumed sample mission requires 170 days for the mission and $31 \mathrm{~kg}$ of xenon mass. If we conduct a longer reorbit mission than 170 days, we could reorbit more numbers of debris objects with the same reorbiter. With a twoyear mission, for example, a simple multiplication indicates that reorbit of more than 24 debris objects is possible. However, there are some constraints on the mission periods. Even if plenty of xenon propellant is loaded, the mission periods would be limited by the ion engine lifetime, and contamination due to back-sputtering.

\section{III.IV Reorbiter System and Ion Engine Arrangements}

The scale of the reorbiter was estimated from the power requirement. The performance of the ion engines assumed in Table 2 requires an ion engine total power of approximately $5.9 \mathrm{~kW}$. From the specifications of past geostationary satellites with similar power levels, the scale of the reorbiter system was estimated as shown in Table 4 . The dry mass of $1240 \mathrm{~kg}$ and the total power of $7 \mathrm{~kW}$ seem within a feasible scale.

We assumed the reorbiter had four thrusters and four backups with $20 \mathrm{mN}$ per thruster for debris irradiation. To raise the orbit of a debris object, these thrusters should be installed on the east panel of the reorbiter. To cancel their reaction, the thrusters for orbit control should be installed on the west panel, and we assumed four thrusters and four backups with $40 \mathrm{mN}$ per thruster. This thrust of $40 \mathrm{mN}$ is the maximum specification value during the descending maneuvers. During the ascending maneuvers, the thrust is throttled because of the limited power and also adjusted to keep a certain separation from the debris object. Figure 4 shows an external view of the reorbiter roughly drawn based on its scale and power.

Instead of installing the clustered thrusters, one single thruster can be used. In this case, one thruster and one backup with $80 \mathrm{mN}$ are necessary on the east panel and one thruster and one backup with $160 \mathrm{mN}$ on the west panel. In the case of using the clustered thrusters, the disturbance torque can be canceled by adjusting the thrust of each 
thruster. In the case of using a single thruster, gimbals are necessary to cancel the disturbance torque, but a simpler configuration can be made.

\section{ION BEAM CONVERGENCE}

\section{IV.I Contrivance for Improving the Ion Beam Convergence}

As described in Section III.I, the improvement in the ion beam convergence is very effective for the reorbiter system. Thus, we contrived two ideas to improve the ion beam convergence. One is to reduce the ion number density in the discharge chamber of the ion thruster. This would allow for a smaller repulsive force among ions in the ion beamlet especially while ions are going through the grid holes. The other idea is to increase the separation between screen and accelerator grids. This would make the equi-potential contours flatter between the grids to allow for less curved ion beam trajectories.

These two ideas are completely contrary to the design policies of ion thrusters for a better performance. In the ion engines for debris irradiation, however, the ion beam convergence precedes the thruster performance. Conventional ion engines have beam divergence angles of about $10 \mathrm{deg}$ or a little larger than that ${ }^{5}$. Making the divergence angles less than that would provide only very small contributions to increasing the thrust, and thus it would be worthless to do so at the cost of degradation in performance. Thus, as far as we know, no one has tried to examine the cases where the beam divergences are much smaller than in the conventional cases. Moreover, we are also interested in the beamlet current contained in circular cones with very small apex angles (3 to 6 deg for example) in addition to the whole beam divergence, which is also a new subject for ion beam optics.

\section{IV.II Numerical Analysis of Ion Beam Trajectories}

A numerical analysis of the ion beam trajectories was conducted to confirm the above ideas to reduce the ion beam divergence and to obtain desirable grid dimensions and operating parameters. We adopted a simple ion extraction system consisting of two flat grids with the center axes of each pair of the grid holes arranged in line, and used a computer code that was developed by one of the authors ${ }^{9}$.

The flowchart of the code and the analyzed region of the ion beam trajectories are shown in Figs. 5 and 6, respectively. First, the electric field in the analyzed region is obtained by solving Poisson's equation, and the ion trajectories in this field are calculated using the equation of ion motion. Then, the ion number density is obtained using conservation of ions. The electron number density is determined by the Boltzmann equation. With these results, the electric field is obtained again. This sequence is repeated until convergence is achieved.

Periodic boundary conditions are applied on the upper and lower boundaries of the analyzed region shown in Fig. 6 , and the potential of $0 \mathrm{~V}$ is applied on the downstream boundary of the analyzed region. The position of the downstream boundary is determined by trial and error. In the numerical analysis, changes of the downstream position in the downstream direction are repeated until almost the same results are obtained, and then the final position in this repetition is treated as the real downstream boundary. Table 5 shows the thruster operating conditions assumed for this analysis.

To examine the effects of the separation between the screen and the accelerator, we calculated the beamlet current, or the beam current per grid hole, within certain divergence angles. Here, the beamlet current within divergence angle $\alpha$ is defined as the beamlet current included in the circular cone with half apex angle $\alpha$, and it is assumed that the ion beam divergence is the same as the beamlet divergence.

The results are shown in Fig. 7 for the divergence angles of 3 and 6 deg. For a discharge chamber ion number density of $3 \times 10^{17} / \mathrm{m}^{3}$, the beamlet current $\mathrm{j}_{\mathrm{b}}$ within 3 deg increased with the increase in the grid separation and reached approximately $30 \mu \mathrm{A}$ for a grid separation of 1.6 to $2.4 \mathrm{~mm}$, while the whole beamlet current itself decreased. 
For an ion number density of $5 \times 10^{17} / \mathrm{m}^{3}$, the situation was almost the same. The effect of the ion number density was not clear. For an ion number density of $5 \times 10^{17} / \mathrm{m}^{3}$, however, ion extraction became impossible for a grid separation larger than $1.5 \mathrm{~mm}$.

Usually, the ion density in the discharge chamber of an ion engine is not uniform but has some radial distribution, which we have to accept when designing ion engine grids. To evaluate the effects of this non-uniformity, we calculated changes in the beamlet currents for a certain range of discharge chamber ion number densities. The results are shown in Fig. 8 for a grid separation of $1.4 \mathrm{~mm}$. The broken curve in Fig. 8 indicates $25 \%$ of the whole beamlet current. For an ion number density range from $2.7 \times 10^{17} / \mathrm{m}^{3}$ to $5.5 \times 10^{17} / \mathrm{m}^{3}$, the beamlet current within a 3 -deg divergence is larger than this broken curve, indicating that it is more than $25 \%$ of the whole beamlet current. It should be noted that the ion engine requirements in Table 2 specify that the half angle containing $25 \%$ of the whole beamlet current be less than $3 \mathrm{deg}$. The results of the calculation suggest that the required grid design is feasible if the nonuniformity of the ion number density in the discharge chamber is within this ion number density range. For each of the grid holes, the beamlet current within a 3 -deg divergence is larger than $25 \%$ of the whole beamlet current. This means that the ion beam current within a 3 -deg divergence is larger than $25 \%$ of the whole ion beam current.

\section{IV.III Experiments on the Ion Beam Convergence}

Experiments on the ion beam convergence were conducted to verify the results of the numerical analysis. For the experiments, we used a ring-cusp type ion thruster with a discharge chamber $10 \mathrm{~cm}$ in diameter, but special model grids were installed, as shown in Table 6, instead of the grids suitable as a conventional thruster. The ion beam was extracted only from the center region of the discharge chamber to achieve uniform beam extraction from the plasma with a certain ion number density.

We made the model grids three times as large as was assumed in the numerical analysis, and thus, to obtain the same value of the ion beamlet current, the ion number density in the experiment should be a ninth of that assumed in the numerical analysis. To keep the similarity of the grid configuration in the numerical analysis and the experiments, all of the grid dimensions were three times enlarged; the hole diameters and thicknesses of the screen and accelerator grids, the grid separation gap and the grid hole pitch.

Divergence measurements were conducted using 31 Faraday cups arrayed along the Y-axis, as shown in Fig. 9. The ion beam was scanned by moving the Faraday cup array along the X-axis to obtain a two-dimensional beam density profile. The two-dimensional beam profiles were measured at several Z-positions to obtain the whole beam structure. To find the beam divergence angle from these data, we processed them as follows. First, we obtained the radial position $r_{\alpha}$ within which $\alpha \%$ of the beam current $J_{b}$ is contained $(\alpha=25,40,60, \ldots)$. Second, the obtained $r_{\alpha}$ data were plotted against the Z-positions. An example plot is shown in Fig. 10. For Z-positions larger than $40 \mathrm{~cm}$, the $r_{\alpha}$-versus- $Z$ contours were almost straight. We finally obtained the divergence half angles from the inclinations of these contours.

For various values of the beamlet current, or the beam current per hole, the divergence measurements were conducted, and the results are shown in Fig. 11. This figure shows that the ion beam divergence angles have a minimum for the beam current per hole of approximately $100 \mu \mathrm{A}$ to $110 \mu \mathrm{A}$. The $25 \%$ divergence half angle was smaller than 3 deg for a beam current per hole of 80 to $130 \mu \mathrm{A}$, and thus, good ion beam convergence was confirmed. The $80 \%$ divergence angle was $6.5 \mathrm{deg}$ at the best case, and it was a little larger than was assumed in the system study.

\section{EFFECTS OF ION BEAM IRRADIATION}

\section{V.I Effects on Debris Objects and the Reorbiter}


This reorbiter system is very unique in that the ion beam irradiates debris objects. This can bring some unique problems because of ion beam irradiation such as thermal effects to the debris objects, ion sputtering of the debris surfaces, and back sputtering to the reorbiter. The first two problems are not critical. In fact, the effects of thermal input due to ion beam irradiation are negligible. It is at much smaller levels than the solar radiation input. Certain levels of ion sputtering of debris surfaces are allowable if its effects are not so large as to generate new debris.

However, back sputtering to the reorbiter may be an important problem because it may cause contamination to the reorbiter system. The back sputtering data of debris surface materials are unavailable as far as we know. Thus, we planned to conduct contamination measurements to obtain back sputtering data.

Table 7 summarizes the outermost materials commonly used for the outer parts of satellites. Among them, glass on solar cells and the polyimide of the MLI occupy large areas of the satellite surfaces, and back-sputtered substances from these materials constitute the dominant contaminant. Thus, we selected these materials for the specimens of these measurements. The surface materials of rocket upper stages also have to be examined, but at present, it is left as a future task.

Back-sputtered substances from a debris object are deposited on the surfaces of the reorbiter that face the debris object. There may be harmful effects to the solar cells (particularly their cover glass), the thermal control materials (typically polyimide film of MLI), and the optical sensors. We worry about degradation of the solar cell performance, changes in the thermal properties of the thermal control materials, and contamination of the optical parts of the sensors. In this research, we examined the contamination of glass and polyimide. If the contamination of the optical parts becomes a serious problem, we can avoid it by using exchangeable filters, for example, but its details are left as a future task.

\section{V.II Contamination Measurements}

Vacuum chamber

Figure 12 shows a schematic of contamination measurements. An ion engine was installed in a vacuum chamber with a beam collector. The ion beam exhausted from the ion engine goes through an aperture opened at the center of the beam collector. Behind the beam collector, we located a set-up for contamination measurements consisting of a beam receiver, a contamination receiver, and a quartz crystal microbalance (QCM). The beam receiver was covered with polyimide film, or alternatively a cover glass, on which a part of the ion beam irradiated. On the contamination receiver, facing the beam receiver, specimens of polyimide film and cover glass were placed to deposit the contaminants on them, and an aluminum mirror was placed to analyze the components of the contaminants with X-ray photoelectron spectroscopy (XPS). The QCM was used to monitor the deposition rates. After a certain time of beam irradiation, these specimens were taken out, and their thermal properties were measured with a spectrophotometer and an emissometer/reflectometer.

The test results of the contamination measurements are shown in Table 8 . The ion beam irradiation time was indicated by the equivalent numbers of reorbits of two-ton debris objects on the assumption that the deposition rate is inversely proportional to the square of the distance from the source. In the sample mission model in Section III.I, the total mass of the six debris objects was $8375 \mathrm{~kg}$. To obtain the same effects as in the sample mission model, the irradiation time should be about four $(8375 / 2000)$ in the above equivalent number. This means that the irradiation time should be 2.2 to 2.8 times longer to have the same quantities of contaminants that would be suffered in the sample mission model.

The results showed that the solar absorptivity $\alpha_{\mathrm{s}}$ and the infrared emissivity $\varepsilon$ of polyimide increased when backsputtered contaminants from glass were deposited. The solar absorptivity $\alpha_{\mathrm{s}}$ of glass increased when the backsputtered contaminants from polyimide were deposited. Except for these three cases, the changes in the thermal properties were negligibly small. 
The transparency data of glass were also obtained in the process of the measurements of the thermal properties. The results are shown in Fig. 13. No changes in the transparency were found when the back-sputtered contaminants from the glass were deposited. However, the transparency was decreased for a shorter wavelength range when the back-sputtered contaminants from polyimide were deposited.

For the case of the deposition of the back-sputtered contaminants from polyimide, the degradation of the solar cells due to the contamination of the cover glass was estimated assuming 10 times the reorbits of two-ton debris objects $18 \mathrm{~m}$ away and cell surfaces facing the sun direction at all times. The results showed that silicon cells and three-junction cells degrade to $94 \%$ and $97 \%$, respectively, of their original efficiencies. The degradation in the threejunction cells is smaller because they utilize the longer wavelength range, in which changes in the transparency is negligible.

The results of the XPS measurements showed that the dominant contaminants were carbon in the case where polyimide was sputtered and silicon dioxide in the case where glass was sputtered.

\section{NON-COOPERATIVE RENDEZVOUS}

Non-cooperative rendezvous is one of the critical technologies that are commonly required in the reorbit of debris objects. At JAXA, we are conducting research on active debris removal in LEO, and we are actively studying noncooperative rendezvous in that research ${ }^{10}$. The results of the research will also be used in non-cooperative rendezvous in the GEO region. It seems that its difficulty level is relatively lower in GEO than in LEO because of the smaller effects of gravity and the more stable optical conditions.

Table 9 summarizes the required measurement sensors for non-cooperative rendezvous according to the distance to a debris object. In long distance rendezvous, the orbit of the debris object is determined by optical observation from ground stations. The capture of the debris object by a long range camera is expected at about a distance of 250 $\mathrm{km}$. An approach up to $10 \mathrm{~km}$ is carried out by repeating the relative distance determinations using a short range camera. An approach up to $100 \mathrm{~m}$ and nearer requires using the camera and also laser sensors. The relative separation maintenance during ion beam irradiation is conducted by navigation using the camera and laser sensors. For the relative separation maintenance, some kind of feed-back control is necessary. A simple simulation indicated that the control should be conducted based on the relative velocity as well as the relative distance.

The application of GPS in the GEO region was studied in the US in the past $t^{11,12}$. We are expecting its realization in the future.

\section{CONCLUSIONS}

We proposed the concept of a reorbiter to reorbit large GEO debris objects by the thrust due to ion beam irradiation. The system study revealed that effective debris reorbit missions can be conducted using this reorbiter system. A beam trajectory analysis and experiments revealed that the required beam convergence is attainable. The measurements of back-sputtering indicated no serious contamination problems. A preliminary study on non-cooperative rendezvous was also conducted.

To realize the reorbiter system, other technical issues have to be addressed in future work. These issues include a detailed study on non-cooperative rendezvous with low thrusting, contamination that may be caused by the backsputtering while irradiating rocket upper stages, and evaluation of the beam divergence using real thrusters. It is also necessary to study if there is a need for chemical attitude control systems to conduct the non-cooperative rendezvous or it can be done completely with electrical propulsion.

\section{REFERENCES}


${ }^{1}$ McKnight, D., "New Insights on the Orbital Debris Collision Hazard at GEO," IAC-11-A6.2.6, 62 ${ }^{\text {nd }}$ International Astronautical Congress, October 2011.

2 Flohrer, T., "Classification of Geosynchronous Objects," GEN-DB-LOG-00086-OPS-GR, Issue 14, February 2012.

3 Starke, J., Bischof, B., Foth, W-H., and Guenther, H-J., "ROGER, a Potential Orbital Space Debris Removal System,” NASA/DARPA International Conference on Orbital Debris Removal, December 2009.

4 Schaub, H., and Moorer, D., “Geosynchronous Large Debris Reorbiter,” NASA/DARPA International Conference on Orbital Debris Removal, December 2009.

5 Kitamura, S., "Large Space Debris Reorbiter Using Ion Beam Irradiation," IAC-10-A6.4.8, 61 ${ }^{\text {st }}$ International Astronautical Congress, September 2010.

${ }^{6}$ Kitamura, S., Hayakawa, Y., and Kawamoto, S., “A Reorbiter for GEO Large Space Debris Using Ion Beam Irradiation,” IEPC-2011-087, 32 ${ }^{\text {nd }}$ International Electric Propulsion Conference, September 2011.

7 Bombardelli, C., Urrutxua, H., Pelaez, J., Merino, M., Ahedo, E., and Summerer, L., "Space Debris Removal with an Ion Beam Shepherd Satellite: Dynamics and Control," IAC-11-A6.5.9, 62 ${ }^{\text {nd }}$ International Astronautical Congress, October 2011.

${ }^{8}$ Bombardelli, C., Merino, M., Ahedo, E., Pelaez, J., Urrutxua, H., Herrera, J., Iturri, A., Olympio, J., Summerer, L., and Petkow, D., "Active Removal of Space Debris - Ion Beam Shepherd for Contactless Debris Removal", European Space Agency, Advanced Concepts Team, Ariadna Final Report (10-4611), July 2011.

9 Hayakawa, Y., "Three-Dimensional Numerical Model of Ion Optics System," Journal of propulsion and Power, Vol. 8, No. 1, 1992, pp. 110-117.

${ }^{10}$ Kawamoto, S., et al., "Control Technologies Required for Electrodynamic Tethers and Active Debris Removal," ISTS-2009-o-4-08v, $27^{\text {th }}$ International Symposium on Space Technology and Science, July 2009.

${ }^{11}$ J. D. Kronman, J., "Experience Using GPS For Orbit Determination of a Geosynchronous Satellite," ION GPS 2000, September 2000.

${ }^{12}$ Ruiz, J., and Frey, C., "Geosynchronous Satellite Use of GPS," ION GNSS $18^{\text {th }}$ International Technical Meeting of the Satellite Division, September 2005. 
Figures

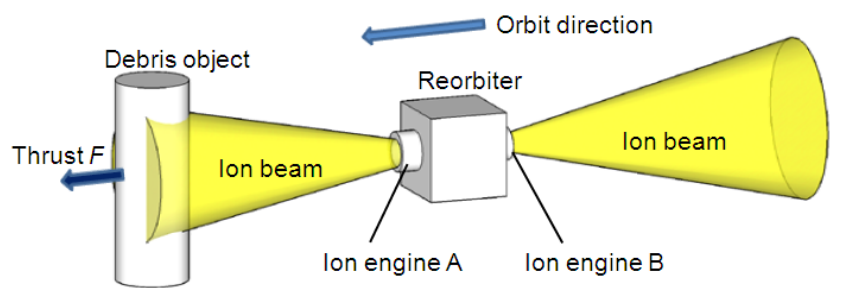

Fig. 1: Schematic drawing of the reorbiter concept.

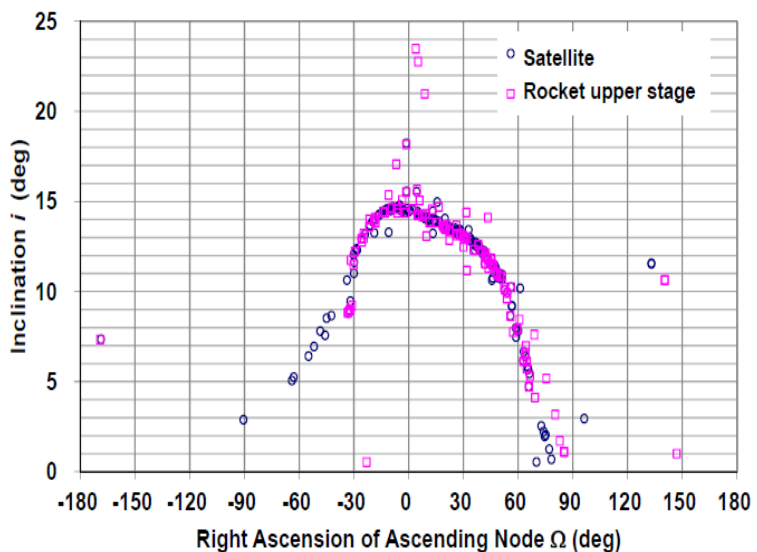

Fig. 2: The orbits of the debris objects in the GEO region on a diagram of the inclination angle $\boldsymbol{i}$ versus the right ascension of ascending node.

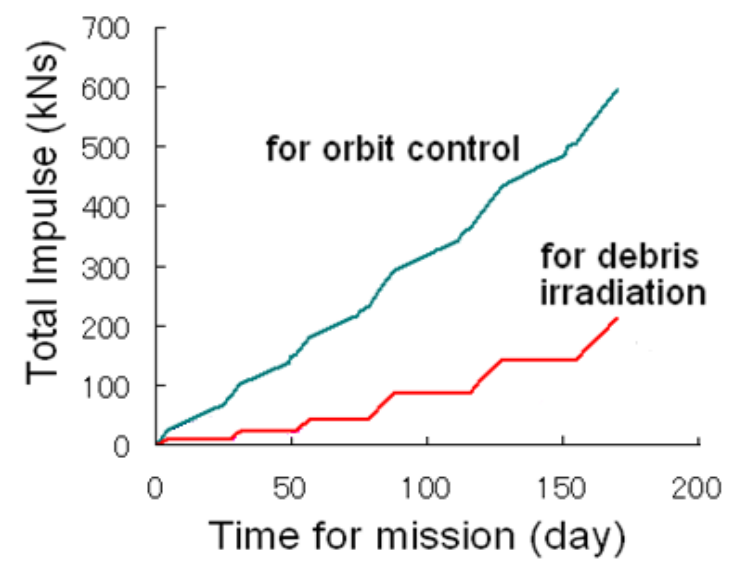

Fig. 3: The total impulse produced by the ion engines for orbit control and for debris irradiation versus the required mission time. 


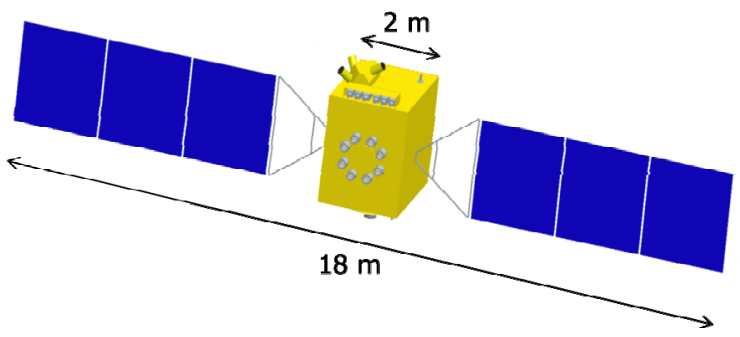

Fig. 4: External view of the reorbiter.

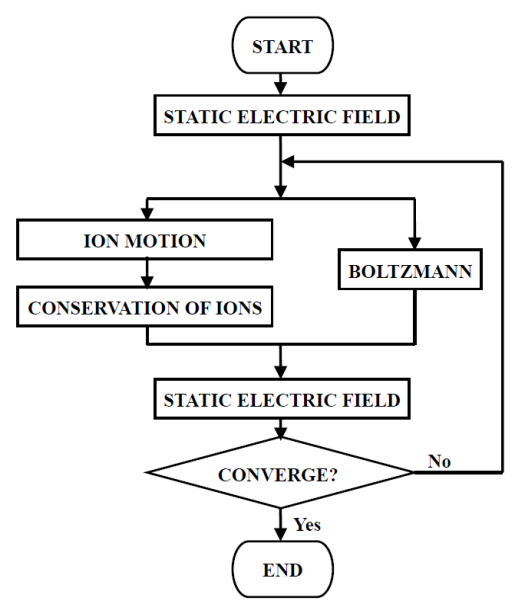

Fig. 5: Flowchart of the computer code for the numerical analysis of ion beam trajectories.

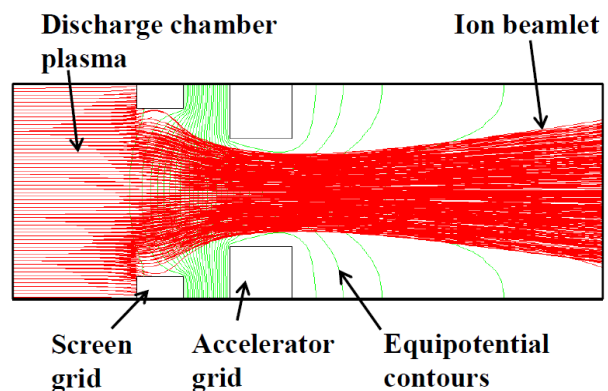

Fig. 6: Analyzed region of the ion beam trajectories. 


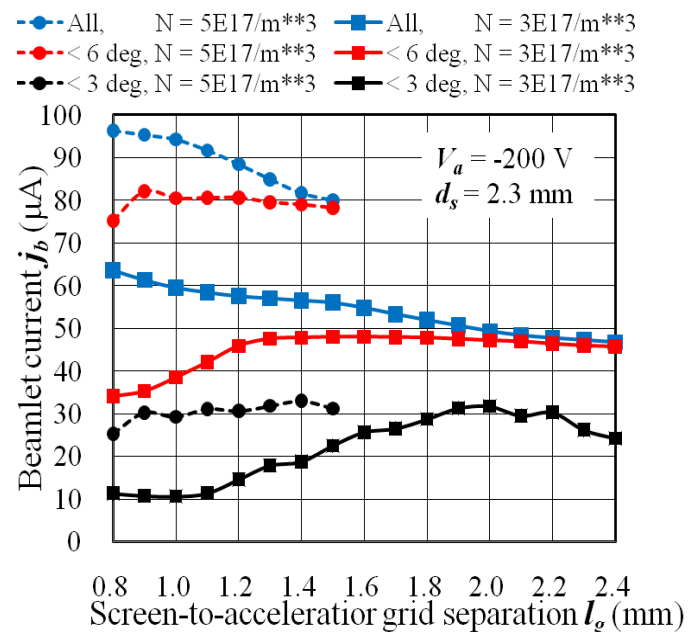

Fig. 7: Beamlet current within certain divergence angles versus the grid gap.

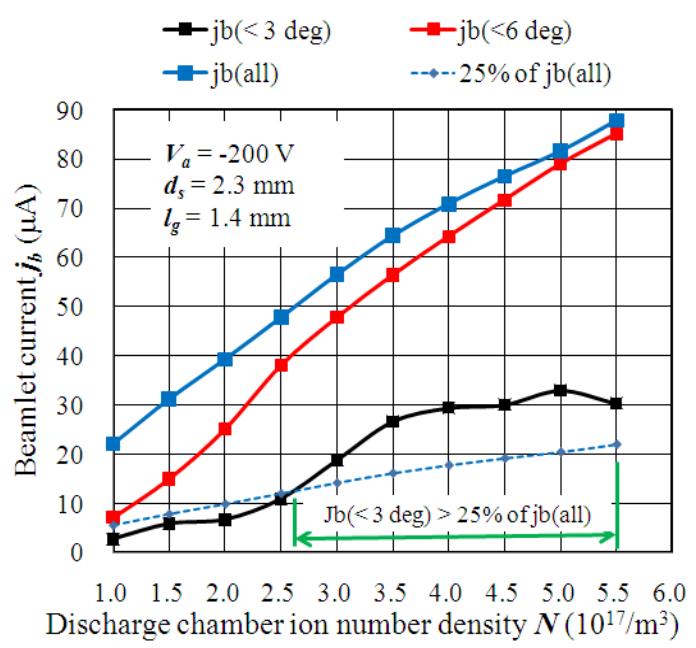

Fig. 8: Beamlet current within certain divergence angles versus the discharge chamber ion number density.

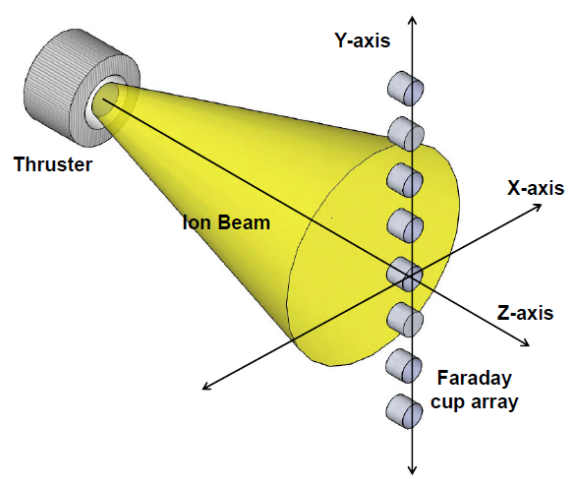

Fig. 9: Layout for the ion beam divergence measurements. 


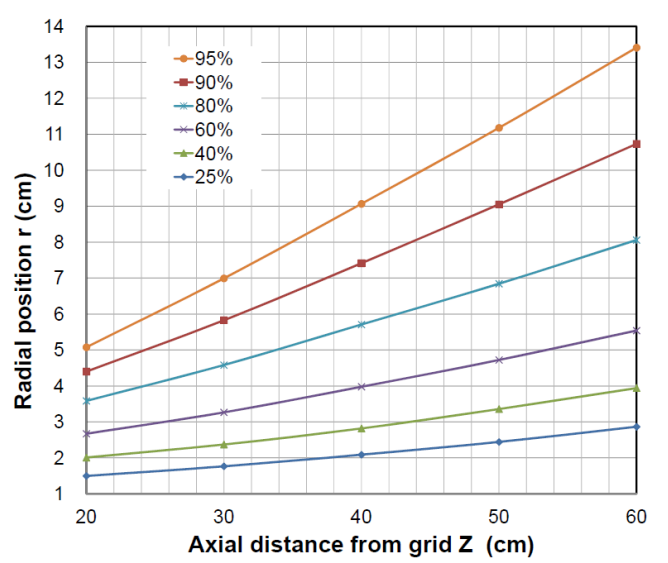

Fig. 10: Example plot of the radial position $r_{\alpha}$ within which $\alpha \%$ of the beamlet current is contained ( $\alpha$ $=25,40,60,80,90$ and 95$)$.

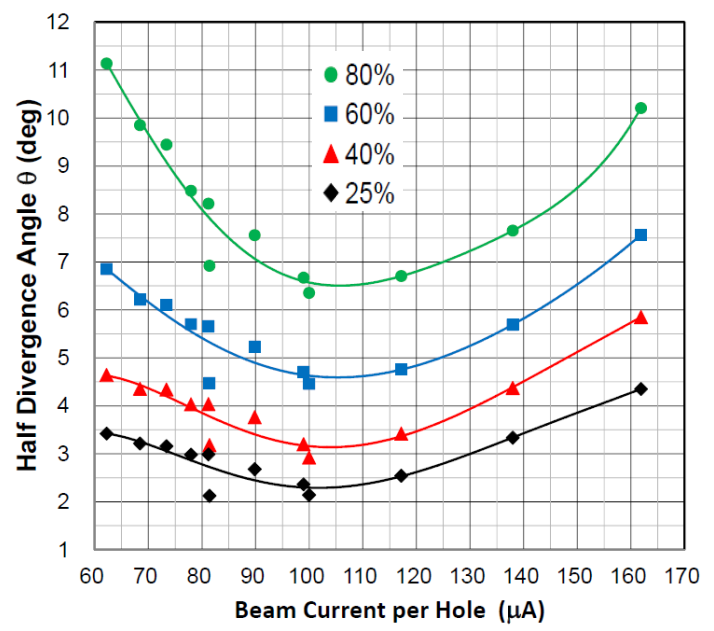

Fig. 11: Divergence angles of $\alpha \%$ of the beamlet current versus the beamlet current ( $\alpha=25,40,60$ and 80$)$. 


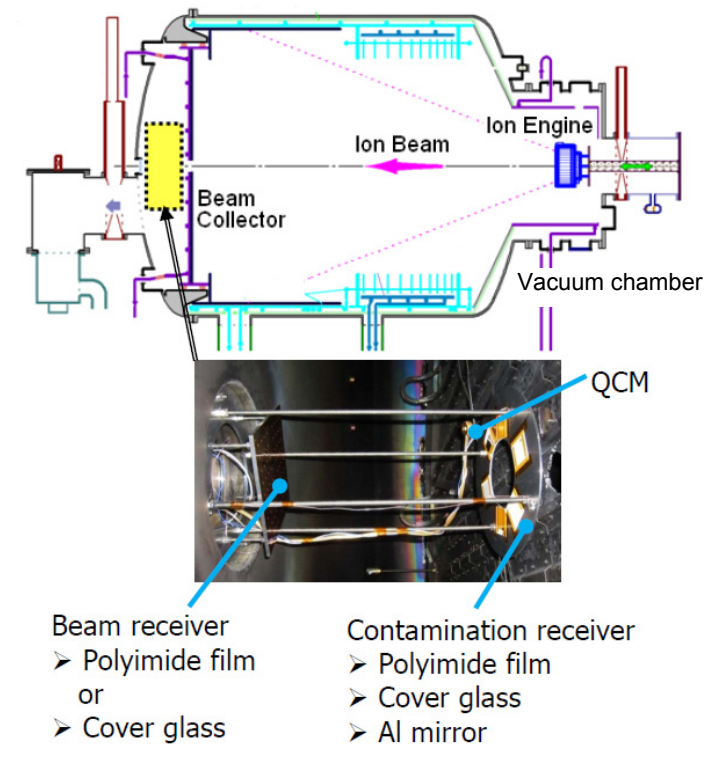

Fig. 12: Schematic of the contamination measurements.

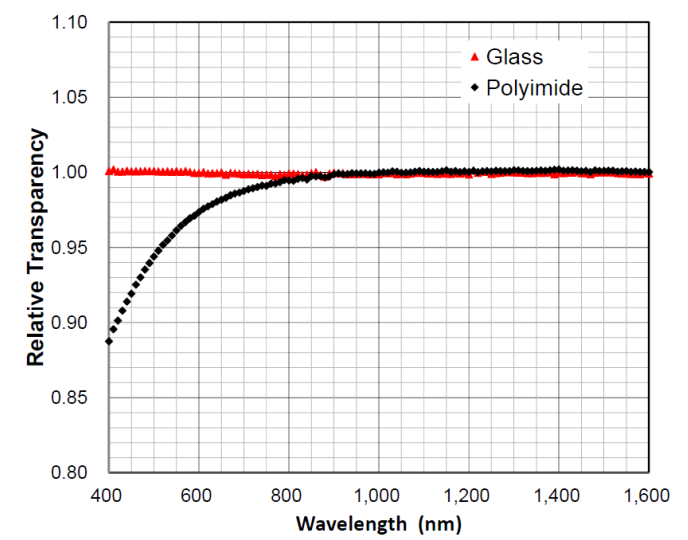

Fig. 13: Changes in the cover glass transparency relative to the new ones. 
Tables

\begin{tabular}{lccccccc}
\hline \multirow{2}{*}{ Debris object } & $\begin{array}{c}\text { Mass } \\
(\mathrm{kg})\end{array}$ & $\begin{array}{c}\text { Diame- } \\
\text { ter } \\
(\mathrm{m})\end{array}$ & $\begin{array}{c}\text { Inclination } \\
(\mathrm{deg})\end{array}$ & $\begin{array}{c}\text { RAAN } \\
(\mathrm{deg})\end{array}$ & $\begin{array}{c}\text { Apogee above } \\
\text { GEO }(\mathrm{km})\end{array}$ & $\begin{array}{c}\text { Perigee above } \\
\text { GEO }(\mathrm{km})\end{array}$ \\
\hline \multirow{3}{*}{ Satellite } & $\# 1$ & 125 & 1.4 & 13.0 & 3 & 0 & 0 \\
& $\# 2$ & 250 & 1.6 & 13.4 & 2 & 125 & 30 \\
& $\# 3$ & 500 & 2.0 & 13.7 & 1 & 125 & -30 \\
\hline \multirow{2}{*}{ Rocket upper } & $\# 1$ & 2000 & 3.0 & 14.0 & 0 & 125 & -125 \\
stage & $\# 2$ & 2500 & 3.7 & 14.5 & -1 & 30 & -250 \\
& $\# 3$ & 3000 & 3.7 & 15.0 & -2 & -30 & -250 \\
\hline
\end{tabular}

Table 1: Sample mission model for reorbit.

\begin{tabular}{|c|c|c|}
\hline Function & $\begin{array}{l}\text { Debris } \\
\text { irradiation }\end{array}$ & $\begin{array}{l}\text { Orbit } \\
\text { control }\end{array}$ \\
\hline $\begin{array}{l}\text { Thrust per } \\
\text { thruster }\end{array}$ & $20 \mathrm{mN}$ & $40 \mathrm{mN}(*)$ \\
\hline Number & $4+4$ backups & $4+4$ backups \\
\hline Specific impulse & $3000 \mathrm{~s}$ & $3000 \mathrm{~s}$ \\
\hline $\begin{array}{l}\text { Thrust-to-power } \\
\text { ratio }\end{array}$ & $25 \mathrm{mN} / \mathrm{kW}$ & $30 \mathrm{mN} / \mathrm{kW}$ \\
\hline Propellant & $\begin{array}{c}\text { Xenon } \\
25 \% \text { half angle }\end{array}$ & Xenon \\
\hline Beam divergence & $\begin{array}{l}<3 \mathrm{deg} \\
80 \% \text { half angle } \\
<6 \mathrm{deg}\end{array}$ & as is \\
\hline
\end{tabular}

Table 2: Ion engine requirements.

\begin{tabular}{clc}
\hline Total impulse & Orbit control & 596 \\
$(\mathrm{kNs})$ & Debris irradiation & 212 \\
& Total & 808 \\
\hline \multirow{2}{*}{ Xenon mass } & Required & 27.5 \\
$(\mathrm{~kg})$ & Margin $(11 \%)$ & 3.5 \\
& Total & 31.0 \\
\hline
\end{tabular}

Table 3: Total impulse and propellant mass required for the sample mission model.

\begin{tabular}{lr}
\hline Dry mass & $1240 \mathrm{~kg}$ \\
\hline Propellant (Xenon) & $31 \mathrm{~kg}$ \\
\hline Beginning-of-mission mass & $1271 \mathrm{~kg}$ \\
\hline Launch mass & $2500 \mathrm{~kg}$ \\
\hline Total power & $7 \mathrm{~kW}$ \\
\hline $\begin{array}{l}\text { Table 4: Mass and power (target) } \text { for the } \\
\text { reorbiter system. }\end{array}$
\end{tabular}




\begin{tabular}{llc}
\hline Beam voltage & $1 \mathrm{kV}$ \\
\hline \multicolumn{2}{l}{ Accel grid voltage } & $-200 \mathrm{~V}$ \\
\hline Discharge voltage & $30 \mathrm{~V}$ \\
\hline Electron & in upstream & $3.0 \mathrm{eV}$ \\
\cline { 2 - 3 } temperature & in downstream & $3.0 \mathrm{eV}$ \\
\hline Propellant & Xenon \\
\hline
\end{tabular}

Table 5: Operating conditions for the numerical analysis of the ion beam trajectories.

\begin{tabular}{lcl}
\hline Scale & 3-fold model & $\begin{array}{l}\text { For exact setting } \\
\text { and measurements }\end{array}$ \\
\hline \# of grids & 2 & Screen and accel \\
\hline \# of holes & 37 in center area & $\begin{array}{l}\text { For uniform ion } \\
\text { number density }\end{array}$ \\
\hline
\end{tabular}

Table 6: Grid system of the thruster for the ion beam divergence measurements.

\begin{tabular}{|c|c|c|c|c|}
\hline \multicolumn{2}{|l|}{ Parts } & \multicolumn{2}{|c|}{$\begin{array}{l}\text { Outermost } \\
\text { material }\end{array}$} & Remarks \\
\hline \multicolumn{2}{|c|}{ Solar cell } & \multicolumn{2}{|l|}{ Glass } & Cover glass \\
\hline \multirow{3}{*}{$\begin{array}{l}\text { Thermal } \\
\text { control } \\
\text { material }\end{array}$} & $\begin{array}{l}\text { MLI (Mul- } \\
\text { ti-layer } \\
\text { insulation) }\end{array}$ & \multicolumn{2}{|c|}{ Polyimide } & $\begin{array}{l}\text { Aluminum- } \\
\text { backed } \\
\text { Carbon- } \\
\text { filled }\end{array}$ \\
\hline & $\begin{array}{l}\text { OSR } \\
\text { (Optical } \\
\text { solar } \\
\text { reflector) }\end{array}$ & \multicolumn{2}{|l|}{ Glass } & $\begin{array}{l}\text { Silver- } \\
\text { coated } \\
\text { quartz glass }\end{array}$ \\
\hline & $\begin{array}{l}\text { Silver } \\
\text { Teflon }\end{array}$ & \multicolumn{2}{|l|}{ PTFE } & \\
\hline \multicolumn{2}{|c|}{$\begin{array}{l}\text { Structure, Back-surface } \\
\text { of solar array, Back- } \\
\text { surface of deployable } \\
\text { antenna }\end{array}$} & \multicolumn{2}{|c|}{$\begin{array}{l}\text { Epoxy } \\
\text { resin, } \\
\text { Carbon } \\
\text { fiber }\end{array}$} & \\
\hline \multicolumn{2}{|c|}{ Fastener, Bracket } & \multicolumn{2}{|c|}{$\begin{array}{l}\text { Aluminum } \\
\text { alloy }\end{array}$} & \\
\hline \multicolumn{2}{|c|}{$\begin{array}{l}\text { Antenna reflector (non- } \\
\text { mesh type) }\end{array}$} & \multicolumn{2}{|c|}{ Paint } & \\
\hline \multicolumn{5}{|c|}{ Table 7: Surface materials of the satellites. } \\
\hline $\begin{array}{l}\text { Base } \\
\text { material }\end{array}$ & $\begin{array}{l}\text { Back- } \\
\text { sputtered } \\
\text { material } \\
\end{array}$ & $\alpha_{\mathrm{s}}$ & $\varepsilon$ & $\begin{array}{l}\text { Irradiation } \\
\text { time* }\end{array}$ \\
\hline \multirow{3}{*}{ Polyimide } & $\mathrm{N} / \mathrm{A}$ & 0.235 & 0.506 & 0 \\
\hline & Polyimide & 0.237 & 0.509 & 1.9 \\
\hline & Glass & 0.252 & 0.519 & 1.5 \\
\hline \multirow{3}{*}{ Glass } & $\mathrm{N} / \mathrm{A}$ & 0.029 & 0.737 & 0 \\
\hline & Polyimide & 0.051 & 0.739 & 1.9 \\
\hline & Glass & 0.030 & 0.737 & 1.5 \\
\hline
\end{tabular}

*Equivalent number of reorbits of 2-ton debris objects 
Table 8: Changes in the solar absorptivity $\alpha_{s}$ and the infrared emissivity $\varepsilon$ resulting from the ion-beam irradiation tests.

\begin{tabular}{|c|c|c|c|c|c|}
\hline & \multirow[b]{2}{*}{$\begin{array}{l}\text { Distance } \\
\text { to debris }\end{array}$} & \multirow[b]{2}{*}{$\begin{array}{l}\text { Ground } \\
\text { support }\end{array}$} & \multicolumn{3}{|c|}{ Measurement sensor } \\
\hline & & & $\begin{array}{l}\text { Long } \\
\text { range } \\
\text { camera }\end{array}$ & $\begin{array}{l}\text { Short } \\
\text { range } \\
\text { camera }\end{array}$ & $\begin{array}{l}\text { Laser } \\
\text { sensor }\end{array}$ \\
\hline $\begin{array}{l}\text { Long } \\
\text { distance } \\
\text { rendez- } \\
\text { vous }\end{array}$ & $>250 \mathrm{~km}$ & X & $(\mathrm{X})$ & - & - \\
\hline \multirow{3}{*}{ Approach } & $\begin{array}{l}10 \mathrm{~km}- \\
250 \mathrm{~km}\end{array}$ & $(X)$ & $X$ & - & - \\
\hline & $\begin{array}{c}100 \mathrm{~m}- \\
10 \mathrm{~km}\end{array}$ & - & $(X)$ & $X$ & - \\
\hline & $\begin{array}{l}20 \mathrm{~m}- \\
100 \mathrm{~m}\end{array}$ & - & - & $X$ & $X$ \\
\hline $\begin{array}{l}\text { Separation } \\
\text { mainte- } \\
\text { nance }\end{array}$ & $20 \mathrm{~m}$ & - & - & X & $X$ \\
\hline
\end{tabular}

Table 9: Applications of the sensors for the navigation system. (X) means auxiliary measures. 